\title{
Repetition blindness: The survival of the grouped
}

\author{
Liat Goldfarb • Anne Treisman
}

Published online: 3 August 2011

(C) Psychonomic Society, Inc. 2011

\begin{abstract}
The repetition blindness (RB) effect demonstrates that people often fail to detect the second presentation of an identical object (e.g., Kanwisher, 1987). Grouping of identical items is a well-documented perceptual phenomenon, and this grouping generally facilitates perception. These two effects pose a puzzle: RB impairs perception, while perceptual grouping improves it. Here, we combined these two effects and studied how they interact. In a series of three experiments, we presented repeated items in a simultaneous string, while manipulating the organization of the repeated items in groups within a string. We observed an interaction between RB and grouping that we summarize with a rule that we call "the survival of the grouped": In essence, the ability to group repeated elements protects them from RB. These findings are discussed within the framework of the object file theory.
\end{abstract}

Keywords Repletion blindness · Grouping · Object files · Perception

An interesting problem for any perceptual system is how to represent identical objects. The answer will probably require a distinction between types and tokens, or between "identification," which depends on the activation of stored types in a recognition network, and "seeing," which results from the creation of temporary episodic tokens or object files. When items are different, they can

L. Goldfarb $(\bowtie) \cdot$ A. Treisman

Department of Psychology, Center for the Study of Brain,

Mind, and Behavior, Princeton University,

Green Hall,

Princeton, NJ 08544, USA

e-mail: 1goldfar@princeton.edu be distinguished by labeling their identities; for example, different type nodes may be activated in a recognition system. But when we have multiple instances of the same identity, representing them requires separate tokens, distinguished by their spatiotemporal locations and their immediate history. Kahneman, Treisman, and Gibbs (1992) proposed the concept of object files, or temporary episodic representations of currently attended objects in their current physical instantiations.

Two perceptual phenomena that raise this issue are repetition blindness and perceptual grouping. With repetition blindness (RB), Kanwisher (1987) showed that people often miss the second presentation of an identical object. She related this to a difficulty in setting up two tokens of the same type within a short interval of time. While RB has obvious costs, in that events may disappear from the environment, it has a corresponding benefit in that it gives priority in perception to new events. (Some have suggested an alternative to the perceptual explanation, explaining RB by memory factors. This view will be discussed more extensively in the General Discussion section.) RB is a robust phenomenon; it can occur with any type of repeated element (e.g., a repeated geometrical shape, letter, or color; Kanwisher, 1987, 1991; Kanwisher, Driver, \& Machado, 1995). The disappearance of a repeated element can be expressed either by a failure to detect it at all, or by a mistaken report of another element instead of the correct one (e.g. Kanwisher, 1987; Kanwisher et al., 1995).

$\mathrm{RB}$ was first shown with sequential presentation, but it soon also became apparent when the stimuli were presented in simultaneous displays. Kanwisher (1991) showed that repeated letters or colors that appeared simultaneously in different locations in space were often missed. With simultaneous presentation, however, perceptual 
grouping becomes a possible variable, and it seems to lead to opposite conclusions from those suggested by RB. Grouping by similarity (usually identity) is a basic perceptual phenomenon (see Rock \& Palmer, 1990, for a review). For example, in Fig. 1 a triangle is perceived, since items of the same color are grouped together. Grouping even governs the level of activation in early visual areas in the brain: Neuroimaging data suggest that items that are grouped together by the similarity principle have higher activation levels than items that cannot be grouped (Beck \& Kastner, 2009). Several studies conducted on brain-damaged patients with right parietal damage have also suggested that grouping may prevent the disappearance of items that would otherwise be invisible because of extinction (e.g., Gilchrist, Humphreys, \& Riddoch, 1996; Ward, Goodrich, \& Driver, 1994). For example, Ward et al. found that the detection rate was improved if the stimulus in the left visual field could be grouped with a stimulus in the right visual field. In these examples, grouping of identical items improves detection, while RB suggests the opposite effect. Perceptual grouping is less salient with sequential or rapid serial visual presentation strings, so our study focused on simultaneous presentations in which we could co-vary repetition and grouping and see how the two interact.

Perceptual grouping clearly depends on spatial arrangement. This can also affect RB. Luo and Caramazza (1996) found that when two identical letters are presented simultaneously with other nonrepeated letters in a circular display, the magnitude of the RB for the identical letters is affected by the number of letters separating them spatially. The RB effect is reduced with no separation between the repeated letters (lag 0 sparing); it is maximal with one-letter separation (lag 1), and then gradually drops off with larger separations. The authors offer an account in terms of type refractoriness, suggesting that the lag 0 sparing is due to an initial partial summation of the effects of the two repeated items followed by a refractory period, which dissipates over time. Note, however, that identical adjacent items are also much more likely to form a perceptual group than identical items separated by intervening items.

Fig. 1 An illustration of the Gestalt principle of grouping by similarity. In this figure, a triangular shape is perceived, since the same color elements are grouped together
De Haan and Rorden (2010) ruled out the possibility that the opposite effects of RB and perceptual grouping depend on the use of different strategies by showing both effects within the same experiment, but they studied RB with identical stimuli and grouping with merely similar stimuli. In the present paper, we used only identical or clearly different stimuli, and we manipulated the spatial grouping of the stimuli. Participants were presented with brief exposures of the critical stimuli, while engaging in a distracting task aimed to decrease the mental resources available for the critical task. We found an interaction between the perceived grouping and the presence or absence of $\mathrm{RB}$, which we summarize with a rule that we call "the survival of the grouped." We will demonstrate how the ability to group a repeated element protects it from RB, preventing its disappearance. In the General Discussion section, we suggest an interpretation within the object file framework.

\section{Experiment 1}

In Experiment 1, strings of color elements were presented and participants were asked to report their colors. Within the strings, the repeated elements appeared either within homogeneously colored groups or spatially intermixed. We predicted that participants should report the items' colors better when the items were organized in groups within a string.

\section{Method}

Participants A group of 6 undergraduate students from Princeton University, with normal or corrected-to-normal vision, participated in the experiment in partial fulfillment of course requirements.

Stimuli The stimulus displays consisted of strings of four colored dots or four colored tilted lines $(\backslash)$ printed in the colors red and green. In each display, the string appeared in the center of a black screen. Two single white digits (1-9) appeared on opposite sides of the string, as part of a distracting task that was added to increase the cognitive load.

All of the colors were formed by the standard ink tool of the program Paint. The size of each character (the digits, the tilted lines, and the dots) was approximately $1.2^{\circ}$. In each string, the colors were either arranged in groups (as in the string red-red-green-green) or alternated (items of the same color were never adjacent, as in the string red-green-red-green). 
Procedure Stimulus presentation and data collection were controlled by a Dell computer with an Intel Xeon central processor. The stimuli were presented on a Dell 19-in. monitor, with a keyboard placed on a table between the participants and the monitor. Participants were run individually. They sat approximately $65 \mathrm{~cm}$ from the computer screen. Participants were asked to perform two tasks: a primary and a secondary task. The primary task was to report the digits' identity, and the secondary task was to report the colors of each of the four items in the string in the order in which they were displayed, from left to right.

Participants pressed the space bar to begin each trial. After 1,300 ms of blank interval, the stimuli appeared for either 68 or $94 \mathrm{~ms}$ randomly, followed by a $200-\mathrm{ms}$ mask created from random lines. Immediately after its disappearance, the participants typed their response to the secondary task on the keyboard. The correct color was typed by pressing the "r" key for the color red and the "g" key for the color green. For each string, participants typed the four items' colors in the order in which the participants thought that they had appeared in the display, from left to right (Color 1 represented the color of the stimulus farthest left, and Color 4 the color of the stimulus farthest right; see Fig. 2). No omissions were allowed. To eliminate from the analysis responses that were merely guesses, participants were asked immediately after they responded to grade their degree of confidence in the string they had typed. They pressed the " 1 " key if they were sure, the " 2 " key if they only thought that their response was correct, and the " 3 " key if they simply guessed. Immediately after the participants responded, another message appeared asking about the identity of the digits that appeared on each side of the display. Participants reported the identity of the digits by pressing the corresponding numbers on the keyboard, typing the identity of the left digit (e.g., "1") before the right digit (e.g., "2"). Then the next trial began. Before the beginning of the experimental block, participants were given 4 trials of practice, and then performed a block of 64 experimental trials.

\section{Results and discussion}

Trials on which participants failed to perform the primary task correctly or on which they stated that they had guessed the answer for the color identity were not included in this analysis (39.8\% of the trials). Of those, $60.0 \%$ were due to an incorrect response to the primary task, and of those, $50.1 \%$ were in the grouped condition. Of the guess responses, $46.2 \%$ were in the grouped condition (thus, there is no suggestion that the number of discarded trials differed between the grouped and the mixed conditions).

For the remaining trials, we calculated for each trial its hit score (a hit was a correct report of a color in its correct location within the string). The mean percentage of hit responses in each condition is shown in Table 1. Paired-samples $t$ tests were applied to these hit rates, with grouped versus mixed display as a within-participants factor. The test revealed a significant effect, $t(5)=3.81$, $p<.02$. The mean hit rates were $89.0 \%$, for grouped colors and $63.5 \%$ for the mixed colors, $t(5)=10.26, p<.01$. (The inclusion of the guess responses did not change the main pattern of results.) In addition, since in the present experiment we used only 6 participants, we also inspected the individual results, which showed that for every participant the grouped condition was reported more accurately than the ungrouped condition.

In two additional analyses, the presentation duration of the stimulus (64 or $94 \mathrm{~ms}$ ) or the stimulus type (dots or tilted lines) was entered as a factor in a two-way analysis of variance that was applied to the mean hit rate, with duration and grouping as within-participants factors in one analysis, and stimulus type and grouping as within-

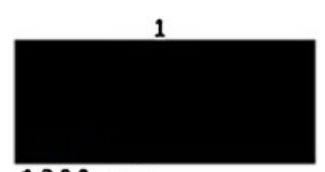

$1300 \mathrm{~ms}$

6

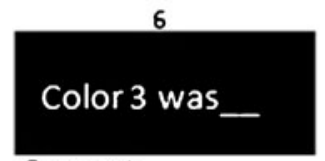

Correct

response: "green"

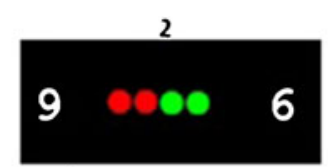

$68 / 94 \mathrm{~ms}$

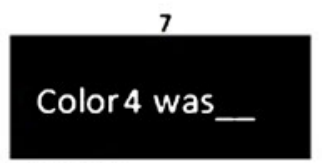

Correct response: "green"

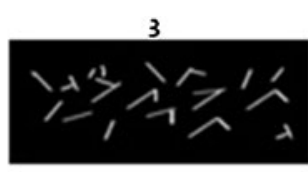

$200 \mathrm{~ms}$

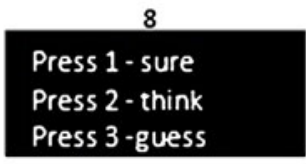

Press 2 - think

Press 3 -guess
Fig. 2 An example that illustrates the trial structure in Experiment 1. The participants were asked to report from left to right the colors of the dots as a secondary task (in this example, red-red-green-green)

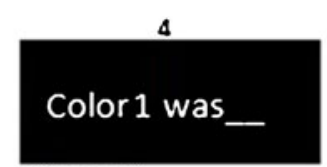

\section{Correct}

response: "red"

9

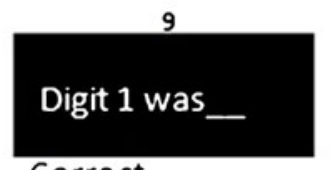

Correct response: "9"

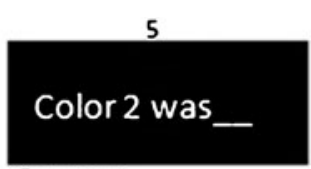

Correct

response: "red"

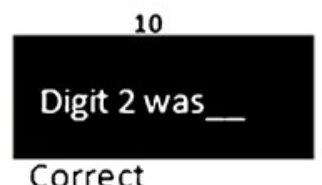

Correct response: " 6 "

and the digits' identity as a primary task (in this example, 9-6) However, only the secondary task was the task of interest 
Table 1 Hit rates as a function of the different conditions in the three experiments

\begin{tabular}{llll}
\hline & $\begin{array}{l}\text { Repeated } \\
\text { Grouped }\end{array}$ & $\begin{array}{l}\text { Repeated } \\
\text { Nongrouped } \\
\text { (Mixed Colors) }\end{array}$ & Nonrepeated \\
\hline Exp. 1 & $89.0(3.6,18.6)$ & $63.5(4.1,19.8)$ & \\
Exp. 2 & $58.2(3.6,19.1)$ & $45.3(3.8,15.5)$ & \\
Exp. 3 & $83.1(3.2,21.0)$ & $59.2(5.7,21.8)$ & $73.6(5.3,24.1)$ \\
\hline
\end{tabular}

In parentheses, the standard errors (SEs) are shown, followed by the mean number of trials per participant.

participants factors in a second analysis. The simple main effect revealed that the grouping effect was significant for the presentation durations of both $64 \mathrm{~ms}, F(1,5)=$ 28.65, $M S E=0.048, p<.01$, and $94 \mathrm{~ms}, F(1,5)=36.29$, $M S E=0.109, p<.001$, as well as for both the dot stimuli, $F(1,5)=35.12, M S E=0.003, p<.001$, and the tilted line stimuli, $F(1,5)=107.30, M S E=0.002, p<.001$. Again, the inspection of the individual results suggested that each of the 6 participants, at each duration or for each presentation type, perceived the grouped condition better than the ungrouped condition. Neither the interaction between duration and grouping nor the interaction between stimulus type and grouping reached significance, $F(1,5)=3.07, M S E=0.1064, p=.13$, and $F(1,5)=3.96$, $M S E=0.002, p=.10$, respectively. Thus, we verified that the grouping effect indeed existed at both presentation durations and with both types of stimuli.

To sum up, the present results suggest that when repeated items are grouped, they are less likely to disappear than when they alternate. In an additional analysis, we examined the extent to which repetition within a group improves rather than impairs detection. The first two items in the grouped strings are identical (e.g., red and red in the strin: red-red-green-green), and the first two items in the mixed strings are different (e.g., red and green in the string red-greenred-green). Would we find the same effect for the first two items in the string as we found for the whole string? To test this notion, a paired-samples $t$ test was applied to the hit rates of the first two items, with grouped versus mixed display as a within-participants factor. The test revealed a significant effect, $t(5)=3.77, p<.02$. The mean hit rates for the detection of the first two items were $92.2 \%(3.1 S E)$ for the grouped condition and $65.7 \%$ (3.6 SE) for the mixed condition, Again, inspection of the individual results suggested that each of the participants showed this effect. Hence, the present experiment suggests that the error rate for repeated items decreases when they are grouped rather than alternating. In fact, in this paradigm a form of alternation blindness replaces repetition blindness.

\section{Experiment 2}

In Experiment 1, there were two fixed variables: the color of items in the display (red and green) and the number of items in the display (four items overall: two red and two green). In addition, the variety of correct responses was limited. The purpose of Experiment 2 was to replicate the effect found in Experiment 1 with a varied number of display items and with a larger variety of correct responses. We predicted that although the overall hit rate would decrease because of the increased heterogeneity, the effect observed in Experiment 1 would not be design specific and should also emerge in other designs.

\section{Method}

Experiment 2 was similar to Experiment 1, except for the following changes: 9 undergraduate students participated in the experiment. The stimulus displays consisted of color dot strings composed of two of the three colors red, green, and blue. The overall number of items in each string was two, three, four, or five, which were equally often displayed. As in Experiment 1, in the three-, four-, and five-dot displays, half of the repeated items were arranged in color groups, and half were intermixed. The two-dot displays were fillers (meant to increase the variety of responses), and they did not contain any repeated colored items. Participants were told the possible numbers of dots, but the strings of different lengths and groupings were randomly intermixed. Omissions were allowed, since the participants were not told for any given string how many items it contained. Each string appeared for $68 \mathrm{~ms}$. Before the beginning of the experimental block, which was composed of 72 trials, participants were given 2 trials for practice.

\section{Results and discussion}

Trials on which participants failed to perform the primary task correctly (naming the digits at either end of the string) or on which they stated that they had guessed the answer for the secondary task were not included in this analysis. (A total of $34.3 \%$ trials were discarded, of which $60.1 \%$ were due to an incorrect response to the primary task; of those, $53.2 \%$ were in the grouped condition. Of the guess responses, $55.5 \%$ were in the grouped condition.) The filler trials (two-item trials) were also not analyzed. As in Experiment 1, the mean percentage of hits in each trial was calculated for the remaining trials for each participant in each condition. The means are shown in Table 1. As in Experiment 1, a 
hit was a correct report of a color in its correct location within the string. A nonhit in this experiment was an item that was omitted from the report, was replaced by another (incorrect) item, or was reported in the wrong location.

Paired-samples $t$ tests were applied to the hit rates, with grouped versus mixed display as a withinparticipants factor. The test revealed a significant effect, $t(8)=3.84, p<.01$. The mean hit rates were $58.2 \%$ for grouped colors and $45.3 \%$ for the mixed colors. [The inclusion of the guess responses did not change the main pattern of results, $t(8)=2.46, p<.05$.] This suggests once again that grouping repeated items resulted in fewer mistakes.

As in Experiment 1, a second analysis was performed to examine the detection rate of the first two items in the grouped and the mixed conditions. A paired-samples $t$ test was applied to the hit rates of the first two colored items, with grouped versus mixed display as a within-participants factor. This analysis was restricted to the four- and five-item displays, since in the three-item displays not all of the items were repeated. As in Experiment 1, the test revealed a significant effect, $t(8)=$ $2.30, p<.05$. The mean hit rates for detection of the first two items were $69.8 \%(4.1 S E)$ for the grouped condition and $55.1 \%$ (5.8 SE) for the mixed condition. Overall, Experiment 2 replicated the findings of Experiment 1 and suggests again that repeated items result in fewer errors when they are grouped together. This inner grouping arrangement also influences the perception of the first two items.

\section{Experiment 3}

In Experiment 3, we examined this effect further in simplified displays. We also compared displays with repeated elements to displays in which no elements were repeated, allowing us to measure $\mathrm{RB}$ as well as the benefit of grouping. Participants observed a string composed of three dots. In the repeated grouped condition, the last dot was grouped with an adjacent dot of the same color (as in the string green-red-red). In the repeated nongrouped condition, the adjacent dot was of a different color (as in the string red-green-red). In the nonrepeated condition, all three dots were different (as in the string blue-green-red). If repeated colors that can be grouped together are indeed protected from RB, then the RB effect should occur only as the difference between the nonrepeated condition and the repeated nongrouped condition, but not between the nonrepeated and the repeated grouped condition.

\section{Method}

Experiment 3 was similar to Experiment 1, except for the following changes: 11 undergraduate students participated in the experiment. The stimulus displays consisted of three colored dot strings composed of either two or three of the following three colors: red, green, and blue. The first two dots in a string were never identical. In $33.3 \%$ of the trials, the last dot in the string was printed in a color that made it a repeated grouped dot (as in the string green-red-red). In another $33.3 \%$ of the trials, the last dot in the string was printed in a color that made it a repeated nongrouped dot (as in the string red-green-red). In the remaining 33.3\% of the trials, the last dot was a nonrepeated dot (as in the string blue-green-red). Each string appeared for $68 \mathrm{~ms}$. Before the beginning of the experimental block, which was composed of 90 trials, participants practiced on 2 trials.

\section{Results and discussion}

Trials on which participants failed to perform the primary task correctly or on which they stated that they had guessed the identity of the color were not included in this analysis. (These criteria excluded $28.6 \%$ of the trials, of which $59.4 \%$ were due to an incorrect response to the primary task; of those, $33.2 \%$ were in the repeated grouped condition, and $33.4 \%$ were in the repeated nongrouped condition. Of the guess responses, $34.5 \%$ were in the repeated grouped condition, and $29.7 \%$ were in the repeated nongrouped condition.)

For the remaining trials, we calculated for each participant in each condition the percentage of correct responses for the last dot, as shown in Table $1 .{ }^{1}$

A one-way analysis of variance was applied to the error rates, with condition (repeated grouped, repeated nongrouped, or nonrepeated) as a within-participants factor. A significant main effect was found for condition, $F(2,20)=11.30, M S E=0.014, p<.001$. [The inclusion of the guess responses did not change the main pattern of results, $F(2,20)=11.39, M S E=0.014, p<.001$.] The correct response rates were $83.1 \%$ for the repeated grouped condition, $59.2 \%$ for the repeated nongrouped condition, and $73.6 \%$ for the nonrepeated condition. The results show both an RB decrement of $14.4 \%$ when no grouping was present [cf. the nonrepeated condition with the repeated nongrouped condition: $F(1,10)=9.38$, $M S E=0.012, p<.01]$, and a large grouping benefit of $23.9 \%$ when repetition was present [repeated grouped minus repeated nongrouped: $F(1,10)=18.62, M S E=$

\footnotetext{
${ }^{1}$ Note that participants reported all three dots, but the critical comparison involved only the last dot. However, performance for reporting all three dots correctly, was very similar to performance on the last dot. The correct response rate was $81.1 \%(0.3 S E)$, for the repeated grouped condition, $63.9 \%(0.5 \mathrm{SE})$, for the repeated nongrouped condition and $74.9 \%(0.5 S E)$ for the nonrepeated condition, $F(2,20)=11.5, M S E=0.012, p<.001$.
} 
$0.016, p<.001]$. The difference of $9.5 \%$ between the repeated grouped and the nonrepeated condition suggests that grouping actually more than eliminated the RB effect; this difference did not quite reach significance but came close: $F(1,10)=3.83, M S E=0.012, p=07$. Overall, this experiment demonstrates that grouping can protect from the RB effect.

Could the difference between the repeated grouped and the repeated nongrouped conditions be explained by a tendency to switch the items or the features between adjacent locations, as might occur in illusory conjunctions? Note that in the repeated grouped condition (e.g., red-green-green), any switch between Locations 2 and 3 cannot be detected as an error. Similarly, in the repeated nongrouped condition (e.g., greenred-green), any switch between Locations 1 and 3 cannot be detected as an error. Accordingly, if there is a tendency to exchange Locations 2 and 3 more than Locations 1 and 3, this tendency could explain why the repeated grouped condition has fewer errors than the repeated nongrouped condition. To address this possibility, we performed the following correction: For the repeated nongrouped condition, we did not classify as errors any responses that switched the colors in Locations 2 and 3. Similarly, for the repeated grouped condition, we did not classify as errors any responses that switched the colors in Locations 1 and 3. This correction allowed us to examine whether a difference between the conditions could still be found when location-switching errors (possible illusory conjunctions) were eliminated. The results revealed that the conditions did still differ. As expected, significantly fewer errors were found for the repeated grouped conditions, $t(10)=2.52, p<.05$. Please note also that our finding of fewer errors in the nonrepeated condition relative to the repeated nongrouped condition is the regular $\mathrm{RB}$ effect, and this cannot be explained by a tendency to switch positions. As noted, when Location 3 switches with Location 1, no error is detected in the repeated nongrouped condition (e.g., green-red-green). However, any type of location switching is considered an error in the nonrepeated condition (e.g., green-red-blue). Hence, according to the switching-position notion, an opposite pattern would be observed (i.e., fewer errors in the repeated nongrouped condition vs. the nonrepeated condition). Accordingly, the observed pattern of results (more errors in the repeated nongrouped condition vs. the nonrepeated condition) must include the notion that repeated features are less discriminable than nonrepeated features, and this notion is the basis of all RB models' assumptions.

\section{General discussion}

This series of experiments clarified the conditions under which repetition impairs performance and under which performance is facilitated. The present experiments were designed to examine the interaction between grouping and $\mathrm{RB}$ when both are processed intentionally. The RB effect clearly suggests that repeated items tend to disappear (e.g., Kanwisher, 1987, 1991). However, we show that grouping can counteract this tendency. In Experiments 1 and 2, repeated items were presented in different orders and participants were asked to report them. These experiments demonstrated that when the repeated items were grouped, they were less likely to disappear than when they were alternated. In fact, identification of the first two items in lists in which the first item was immediately repeated (e.g., red and red in the string redred-green-green) was easier than identification of the first two items in an alternating list (e.g., red and green in the string red-green-red-green). We can think of this as a form of alternation blindness. In Experiment 3, we extended these findings by demonstrating that grouping can completely protect repeated items from the RB effect. These three experiments demonstrated the principle that we call "the survival of the grouped," in which even a repeated element can be protected from potential disappearance by grouping with other nonunique features. Only when there is no "force of the whole" to hold repeated elements together will they tend to disappear.

Luo and Caramazza (1996) suggested that the lag 0 sparing in $\mathrm{RB}$ with simultaneous presentations was due to an initial partial summation of the effects of the two repeated items on the corresponding type node, followed by a refractory period, which dissipates over time. It is not clear that their model can account for the complete abolition, indeed the reversal, of $\mathrm{RB}$ that we found with adjacent items. We suggest that the fact that their items were presented on the arc of a circle may have meant that adjacent items were less likely to group together than our aligned elements. We attribute the two arms of the U-shaped function to two separate causes-grouping of elements at lag 0 , and $\mathrm{RB}$ or failure to form a separate token for the repeated item when intervening items are present.

As we already described, a common explanation for the RB effect is a perceptual deficiency. However, some researchers suggest that this effect can be better explained as a memory deficiency. RB is then equated with a form of the Ranschburg effect, which suggests that in a serial memory recall task it is harder to recall a repeated item than a new item. According to this view, participants have no problem perceiving repeated items, but they have difficulties in retrieving them, either because they are biased against reporting repeated items (e.g., Fagot \& Pashler, 1995) or because of response competition at the retrieval stage (e.g., Armstrong \& Mewhort, 1995). Memory or retrieval problems may play 
a part in some experiments, but they seem unlikely to explain our results. We demonstrate effects under conditions in which both the repeated grouped items and the repeated ungrouped items form unique and easily remembered patterns. For example, the string red-green-red creates as unique a pattern as the string red-green-green, yet the present study suggests that the first is subject to the RB effect, while the second is not.

There are also more specific differences making the parallel with the Ranschburg effect unlikely. The Ranschburg effect is seen in serial memory tasks and applies neither to repeated items that appear at lag 0 (as in our repeated grouped condition) nor to those that appear at lag 1 (as in our repeated ungrouped condition; see Crowder, 1968). In Crowder's study, the ability to recall a repeated item at lag 0 was identical to the ability to recall one at lag 1 , and both were superior to the recall of a new item. In a more recent memory task using presentations similar to those in the present study (simultaneous presentation immediately followed by a mask), no recall deficiency was found for repeated digits at lag 1 relative to new items (Jahnke \& Bower, 1986). Hence, although it is possible that some of the RB effects reported in the literature have a memory component to them, our present results seem to describe a phenomenon that differs from the Ranschburg memory effect.

As we noted, our results suggest that only when there is no "force of the whole" to hold repeated elements together do they tend to disappear perceptually. But what is this "force of the whole," and how in particular can it protect repeated elements from disappearing? Object file theory may provide a useful framework to explain this finding. The theory (Kahneman et al., 1992) claims that in order for us to perceive objects consciously, their features must be bound to locations and to each other within object representations. Each representation must be individuated for us to perceive it as a distinct object. Object file theory distinguishes between types and temporary episodic tokens or object files. To allow the perception of multiple identical objects, all of which would activate the same single type node in a recognition network, object representations must distinguish different individual instances that are simultaneously present, even when they are otherwise identical.

Within the object file framework, we can interpret the interaction between $\mathrm{RB}$ and grouping that was found in the present study. The hypothesis is that RB does not occur between items that belong to the same object file. Grouping by color similarity is one principle (among many others) under which object files can be created. When grouped items are perceived as one object that contains multiple elements, the system simply identifies the grouped elements and the feature information once, for all of the elements together. For example, when one sees five dots in the order red, red, green, green, green, according to the gestalt rule of similarity, two object files may be created. The first file contains two dots, and the second file contains three dots. The relevant colors can then be entered into each file for all of the dots that they contain (red for File 1, and green for File 2). In this case, the repeated colors within the file are protected from the effects of repetition. They are, if anything, easier to see than nonrepeated items, which would tend to require multiple object files.

Note that our claim is not that each object file can contain only a single color. If elements can be grouped by some other feature, a single multicolored object may be seen. There is considerable flexibility in selecting what should be entered into a single object file-for example, the level may shift from a nose, to a face, or to a person, depending on what the perceiver is currently attending to. But grouping by similarity is likely to be one factor that segregates potential object files, and once that has played a role, the principle that $\mathrm{RB}$ does not apply within object files may help to explain our findings.

Some recent neurological evidence is consistent with this object file framework: $\mathrm{Xu}$ and Chun (2007) distinguished the roles in visual working memory of two brain areas. They found that grouped shapes produced less activation than ungrouped shapes in the inferior intraparietal sulcus (IPS), but not in the superior IPS. They suggested that inferior IPS individuates object files and that superior IPS is in charge of entering and updating feature information and identifying the objects.

The object file framework is consistent with a variety of RB models, and particularly with models that attribute the effect to a failure to detect a repeated item. For example, it is consistent with the idea that the repeated item disappears because it fails to be individuated as a separate token (e.g., Kanwisher, 1987). It is also consistent with models that attribute the disappearance of a repeated item to low activation caused by a refractory period (Luo \& Caramazza, 1995, 1996). It also fits with models that assume that masking from the different items in the display causes the repeated item to disappear (e.g., Morris, Still, \& Caldwell-Harris, 2009). Finally, it fits with models that assume that the perceptual construction of repeated items is damaged, where construction includes the attachment of an event to its context (e.g., Masson, Caldwell, \& Whittlesea, 2000; Whittlesea \& Hughes, 2005). Regardless of the specific source that causes the repeated item to disappear, we believe that grouping converts the repeated items into a single perceptual unit, and thereby eliminates the perceptual problem associated with repetition. 
Author Note This research was supported by fellowships from the Israel Science Foundation (Bikura), the Rothschild Foundation, and the Weizmann Institute of Science-Advancing Women in Science (to L.G.) and by NIH Grants 2RO1MH058383, on visual coding and the deployment of attention, and 1RO1MH062331, on spatial representations and attention (to A.T.).

\section{References}

Armstrong, I. T., \& Mewhort, D. J. K. (1995). Repetition deficit in rapid-serial-visual-presentation displays: Encoding failure or retrieval failure? Journal of Experimental Psychology: Human Perception and Performance, 21, 1044-1052. doi:10.1037/00961523.21.5.1044

Beck, D. M., \& Kastner, S. (2009). Top-down and bottom-up mechanisms in biasing competition in the human brain. Vision Research, 49, 1154-1165. doi:10.1016/j.visres.2008. 07.012

Crowder, R. G. (1968). Intraserial repetition effects in immediate memory. Journal of Verbal Learning and Verbal Behavior, 7, 446451. doi:10.1016/S0022-5371(68)80031-3

de Haan, B., \& Rorden, C. (2010). Similarity grouping and repetition blindness are both influenced by attention. Frontiers in Human Neuroscience, 4, 20. doi:10.3389/fnhum. 2010.00020

Fagot, C., \& Pashler, H. (1995). Repetition blindness: Perception or memory failure? Journal of Experimental Psychology: Human Perception and Performance, 21, 275-292. doi:10.1037/00961523.21.2.275

Gilchrist, I. D., Humphreys, G. W., \& Riddoch, M. J. (1996). Grouping and extinction: Evidence for low-level modulation of visual selection. Cognitive Neuropsychology, 13, 1223-1249. doi: $10.1080 / 026432996381737$

Jahnke, J. C., \& Bower, R. E. (1986). Are there two repetition effects? American Journal of Psychology, 99, 275-288

Kahneman, D., Treisman, A., \& Gibbs, B. J. (1992). The reviewing of object files: Object-specific integration of information. Cognitive Psychology, 24, 175-219. doi:10.1016/ 0010-0285(92)90007-O
Kanwisher, N. G. (1987). Repetition blindness: Type recognition without token individuation. Cognition, 27, 117-143. doi:10.1016/0010-0277(87)90016-3

Kanwisher, N. G. (1991). Repetition blindness and illusory conjunctions: Errors in binding visual types with visual tokens. Journal of Experimental Psychology: Human Perception and Performance, 17, 404 421. doi:10.1037/0096-1523.17.2.404

Kanwisher, N., Driver, J., \& Machado, L. (1995). Spatial repetition blindness is modulated by selective attention to color or shape. Cognitive Psychology, 29, 303-337. doi:10.1006/cogp.1995.1017

Luo, C. R., \& Caramazza, A. (1995). Repetition blindness under minimum memory load: Effects of spatial and temporal proximity and the encoding effectiveness of the first item. Perception \& Psychophysics, 57, 1053-1064. doi:10.3758/BF03205464

Luo, C. R., \& Caramazza, A. (1996). Temporal and spatial repetition blindness: Effects of presentation mode and repetition lag on the perception of repeated items. Journal of Experimental Psychology: Human Perception and Performance, 22, 95-113. doi:10.1037/ 0096-1523.22.1.95

Masson, M. E. J., Caldwell, J. I., \& Whittlesea, B. W. A. (2000). When lust is lost: Orthographic similarity effects in the encoding and reconstruction of rapidly presented word lists. Journal of Experimental Psychology: Learning, Memory, and Cognition, 26, 1005-1022. doi:10.1037/0278-7393.26.4.1005

Morris, A. L., Still, M. L., \& Caldwell-Harris, C. L. (2009). Repetition blindness: An emergent property of inter-item competition. Cognitive Psychology, 58, 338-375. doi:10.1016/ j.cogpsych.2008.08.001

Rock, I., \& Palmer, S. (1990). The legacy of Gestalt psychology. Scientific American, 263, 84-90.

Ward, R., Goodrich, S., \& Driver, J. (1994). Grouping reduces visual extinction: Neuropsychological evidence for weight-linkage in visual selection. Visual Cognition, 1, 101-129. doi:10.1080/ 13506289408402295

Whittlesea, B. W. A., \& Hughes, A. D. (2005). The devil is in the detail: A constructionist account of repetition blindness. In N. Ohta, C. M. MacLeod, \& B. Uttl (Eds.), Dynamic cognitive processes (pp. 101-130). Tokyo: Springer.

$\mathrm{Xu}, \mathrm{Y} .$, \& Chun, M. M. (2007). Visual grouping in human parietal cortex. Proceedings of the National Academy of Sciences, 104, 18766-18771. doi:10.1073/pnas.0705618104 